\title{
Das naturphilosophische Konzept der Physiologie bei Johann Christian Friedrich Harless*
}

\author{
Von Charlotte Triebel-Schubert
}

Es ist kein Zufall, daß auffallend viele Anhänger der Schellingschen Naturphilosophie 1818 an die neugegründete Bonner Rheinuniversität berufen wurden. ${ }^{1}$ Die Ursache liegt in den guten persönlichen Beziehungen David Koreffs zu dem preußischen Kanzler Hardenberg, der Koreff aus diesem Grund mit den Bonner Berufungsangelegenheiten betraute. ${ }^{2}$ Koreff war neben Karl Wolfart der führende Berliner Vertreter des tierischen Magnetismus, einer therapeutischen Konzeption, deren Affinität zur romantischen und auch zur naturphilosophisch orientierten Medizin nahelag. So bevorzugte Koreff bei seinen Vorschlägen erklärte Anhänger der Naturphilosophie. Während man sich um F. W. J. Schelling selbst und D. G. Kieser vergeblich bemühte, konnte man aber doch andere namhafte Vertreter dieser Richtung gewinnen: Friedrich Nees von Esenbeck, Carl Gustav Bischof, Ernst Bischof, Christian Brandis, Ph.F.v. Walther, Karl Windischmann und Johann Christian Friedrich Harless.

Gerade Harless, der bis dahin ausschließlich in Erlangen gelehrt hatte, ${ }^{3}$ muß als ein typischer und außerordentlich konsequenter Vertreter der Schellingschen Lehre bezeichnet werden. ${ }^{4}$ Harless übertrug die Sätze der Schellingschen Philosphie mit einer Folgerichtigkeit und Direktheit auf das Gebiet der Physiologie und auch auf das der Pathologie, die auffallend ist und ihn so auch aus der Menge der philosophierenden Ärzte dieser Zeit heraushebt.

Harless selbst charakterisiert seinen eigenen theoretischen Standpunkt allgemein als den eines kritischen Eklektizismus. ${ }^{5}$ Aus diesem Grund wurde er bisher auch nicht zu den Vertretern der naturphilosophischen Medizin gerechnet, sogar eher ihren Kritikern zugeordnet. ${ }^{6}$ Andererseits findet sich in seiner Definition eines solchen kritischen Eklektizismus ein relativ klares Bekenntnis zur Naturphilosophie: «Aber er (der Arzt) schöpft diese (allgemeine und durch geistige Abstraktion geschöpfte Prinzipien) nicht auf dem

\footnotetext{
* Umgearbeitetes Manuskript eines Vortrages, den ich am 20.11.1982 in Münster anläßlich eines Fortbildungsseminars zu dem Thema «Medizin und Philosophie im 19. Jahrhundert» gehalten habe.
} 
unmittelbaren Boden einer empirischen Naturlehre und Medicin, in deren Grenzen sie nicht liegen können. Er entnimmt sie aus den gränzenlosen Räumen der uneigentlich sogenannten metaphysischen Speculation, aus dem Reich der Ideen und Hypothesen ... (Rep. 32).

Im Einzelnen läßt sich dies an seinen methodischen Vorstellungen, seinem System der Physiologie und der daraus abgeleiteten Theorie der Krankheit belegen.

\section{Methodische Vorstellungen}

Harless unterlegt seinem kritischen Eklektizismus in der Medizin ein dreistufiges Erkenntnismodell: ${ }^{7}$

1.Stufe: reine Empirie

2.Stufe: medizinische Theorie - Philosophie

3. Stufe: reine, absolute Erfahrung

Unter der reinen Empirie, ${ }^{8}$ der ersten und einfachsten Stufe der Erkenntnis, versteht Harless ein bestimmtes Verhalten des Arztes. In der Therapie bedeutet die reine Empirie das Handeln am Krankenbett nach Versuchsresultaten, das Vergleichen von Kurarten und Heilmitteln ohne das Erforschen eines inneren Handlungsgrundes. Ebenso definiert er die angemessene Funktion der Empirie in der speziellen Pathologie als ein einfaches Zusammenstellen der Erscheinungen ohne solchen Beobachtungen ein höheres Prinzip zugrunde zu legen. Als höchste Form dieser Empirie bezeichnet er eine Erfahrung im empirischen Sinn, eine «veredelte Empirie», die sich jedoch qualitativ nicht über die erste Stufe erhebt. Besonderer Angriffspunkt ist für ihn in diesem Zusammenhang die Richtung des Empirismus, für den Empirie nicht nur Mittel, sondern auch alleiniger Zweck und alleiniges Ziel ist.

Nach Harless' Vorstellungen steht dieser ersten, einfachen Stufe der Empirie eine zweite gegenüber, deren Inhalt die Verbindung von medizinischer Theorie und Philosophie ist. Die Medizin bestimmt innerhalb dieser zweiten Stufe Grenzen und Zweck von Theorie und Spekulation, basiert jedoch auf den Grundsätzen der allgemeinen Physiologie. Die Physiologie wiederum ist Teil der spekulativen Naturphilosophie und nimmt aus ihr die konstitutiven Grundsätze. ${ }^{9}$

Nach der ersten einfach-empirischen und der zweiten, theoretisch-spekulativen Stufe definiert Harless noch eine dritte, die der reinen, absoluten 
Erfahrung. Sie ist die Synthese aus reiner Empirie und spekulativer Theorie der Medizin. ${ }^{10}$ Deuten neben der Verwendung der dialektischen Triade zur Konstruktion seines Erkenntnismodelles auch die inhaltlichen Bestimmungen auf die Position eines Naturphilosophen, so läßt sich dies ebenso an seinem System der Physiologie erkennen.

\section{System der Physiologie}

Das System der Physiologie, ${ }^{11}$ das Harless in seinem ersten Band des Handbuches der Klinik entwickelt, ist ebenso wie die gesamte organische Natur bestimmt durch die dynamische Urpolarität eines expansiven und eines kontraktiven Urprinzipes. Aus diesen beiden Tätigkeitsprinzipien, denen er die beiden Urkräfte (positiv - negativ) entsprechen läßt, deduziert er ganz parallel zu Schelling die Konstruktion der Materie. ${ }^{12}$ Die wechselnden Verhältnisse der expansiven und kontraktiven Urkräfte zueinander entsprechen den Faktoren zur Konstruktion dieser Materie. ${ }^{13}$ Die "physische Raumerfüllung nach ihrem objektiven Ausdruck oder nach ihrer Durchbildung in ein Räumliches und Gestaltbares ist Materie ...». Die Entwicklung zur physischen Raumerfüllung entspricht der Deduktion der drei Dimensionen der Materie bei Schelling, wobei Schelling die folgenden Entsprechungen für die einzelnen Dimensionen sieht:

1. Dimension: Länge-Magnetismus

2. Dimension: Breite-Elektrizität

3. Dimension: Tiefe-chemischer Prozeß. ${ }^{14}$

Damit sind auch die drei Stufen des dynamischen Prozesses gegeben. Auch Harless entwickelt den dynamischen Prozeß aus der Konstruktion der Materie: Denn diese ist bei ihm durch die sich wiederum manifestierende Bipolarität von Lichtstoff und Erdstoff gekennzeichnet ${ }^{15}$ (negativ/expansiv - positiv/kontraktiv). Sie prägt den dynamischen Prozeß der Entwicklung von organischem Leben in dessen vielfältigen Abstufungen. ${ }^{16}$ Schelling hat mit der Übertragung des Bildes von der galvanischen Reaktion in die Philosophie einen allgemeinen Ausdruck dieses in das Produkt übergehenden dynamischen Prozesses gefunden. Auch bei Harless symbolisiert das Bild der galvanischen Reaktion das Grundgesetz des Lebensprozesses: Die Bildung des Organismus (= Produkt) ist das Ergebnis des polaren Verhältnisses der beiden Grundkräfte (= Prozeß). So leitet er daraus mit Hilfe der dialektischen Triplizität drei organische Systeme ab: ${ }^{17}$ 

1. Irritabilitätssystem:
entsprechend der positiven, kontraktiven Urkraft
2. Sensibilitätssystem: entsprechend der negativen, expansiven Urkraft
3. Plastizitätssystem:
entsprechend der dritten, aus beiden polaren Urkräften zusam- mengesetzten Kraft

Hierbei ist zu betonen, daß in dem ersten und zweiten System die genannte Grundkraft nur die überwiegende, nicht die alleinig vorhandene ist, und daß auf Grund der durchgängigen Polarität beide Grundkräfte nicht nur zwischen den Systemen, sondern auch innerhalb eines Systems in ihrem Antagonismus wirksam sind. So ist Harless' System der Physiologie durch eine ständige Wechselwirkung zwischen den und innerhalb der einzelnen Stufen gekennzeichnet.

Bemerkenswert ist, daß Harless das dritte System zwar als das aus den beiden anderen zusammengesetzte beschreibt, es in der Stufenfolge der Systeme jedoch als die niedrigste Stufe in der organischen Bildung einordnet, als diejenige der einfachen Reproduktion. ${ }^{18}$

Die sich bei Harless ergebende Stufenfolge der Systeme, Reproduktion bzw. Plastizität - Irritabilität - Sensibilität, ist formal die gleiche, die Schelling seit 1805, erstmals schon 1803 in den Vorlesungen über die Methode des akademischen Studiums angedeutet, vertrat. ${ }^{19} \mathrm{Im}$ Gegensatz zu der ursprünglichen Reihenfolge, wie Schelling sie in dem Ersten Entwurf eines Systems der Naturphilosophie (1799) aufgestellt hatte (Sensibilität Irritabilität - Bildungstrieb/Reproduktionskraft), ${ }^{20}$ kehrte er später Sensibilität und Reproduktion in ihrem Platz innerhalb der dialektischen Triade um. ${ }^{21}$ Entscheidend blieb jedoch immer, daß die dritte Potenz als Synthese der Polarität von erster und zweiter Potenz auch innerhalb des dynamischen Prozesses des Organismus als der vollkommenste organische Zustand ausgedrückt wurde. Schelling bezeichnete diesen Zustand als die «Idee eines Gleichgewichtes der Empfänglichkeit und Thätigkeit» ${ }^{22}$. Ein durchaus vergleichbares Zitat bei Harless zur Plastizitäts- bzw. Reproduktionsstufe lautet:

«Die Stufe (zugleich Klasse) des organisch-plastischen und reproduktiven Lebens gehet hervor aus dem einfachsten und mehr oder weniger dem Gleichgewicht (chemisch ausgedrückt, der Neutralisierung) sich nähernden Kombinationsverhältnis von reiner (elementarer) Sensibilität und Irritabilität.» (Handb. I 63)

Harless fährt dann jedoch folgendermaßen fort:

«Sie ist die niedrigste aus der Scale des Lebens und der Organisation, und gränzt am nächsten an das Reich des Unorganischen, das ebenfalls nur durch 
einen successiven Übergang vorwärts (so wenigstens immer gattungsweise) sich an sie reiht.»

Schelling legte schon zu Anfang eine Entwicklung von den einfachen zu den zusammengesetzten Kräften zugrunde, indem er in seiner Materiededuktion die Schwere als die aus Repulsion und Attraktion zusammengesetzte Kraft ableitet:

«Die Schwerkraft selbst ist nicht identisch mit der Attraktivkraft, obgleich diese nothwendig in sie mit eingeht. Die Schwerkraft ist auch nicht eine einfache Kraft, wie diese, sondern, wie aus der Deduktion erhellt, eine zusammengesetzte Kraft.» (Syst. d.tranz. Id. a. O. 444)

Dieselbe Entwicklung von den einfachen zu den zusammengesetzten Tätigkeiten liegt bei Harless der Ableitung der drei organischen Systeme zugrunde, denn auch er benutzt das Prinzip der Triplizität, versinnbildlicht im galvanischen Prozeß, als das allgemeine Gesetz des Lebensprozesses.

Trotzdem weist die Stellung des Plastizitätssystemes in der Harlessschen Konzeption der Physiologie eine besondere Eigentümlichkeit auf:

Obwohl es als ein zusammengesetztes System der Synthesis der dialektischen Triade entspricht, wird es den aus den dualistischen Urprinzipien abgeleiteten, nicht zusammengesetzten Systemes untergeordnet, so daß sich folgendes Schema seines physiologischen Systems aufstellen läßt: ${ }^{24}$

Dynamische Grundverhältnisse des Lebens, v. a. des tierischen Organismus

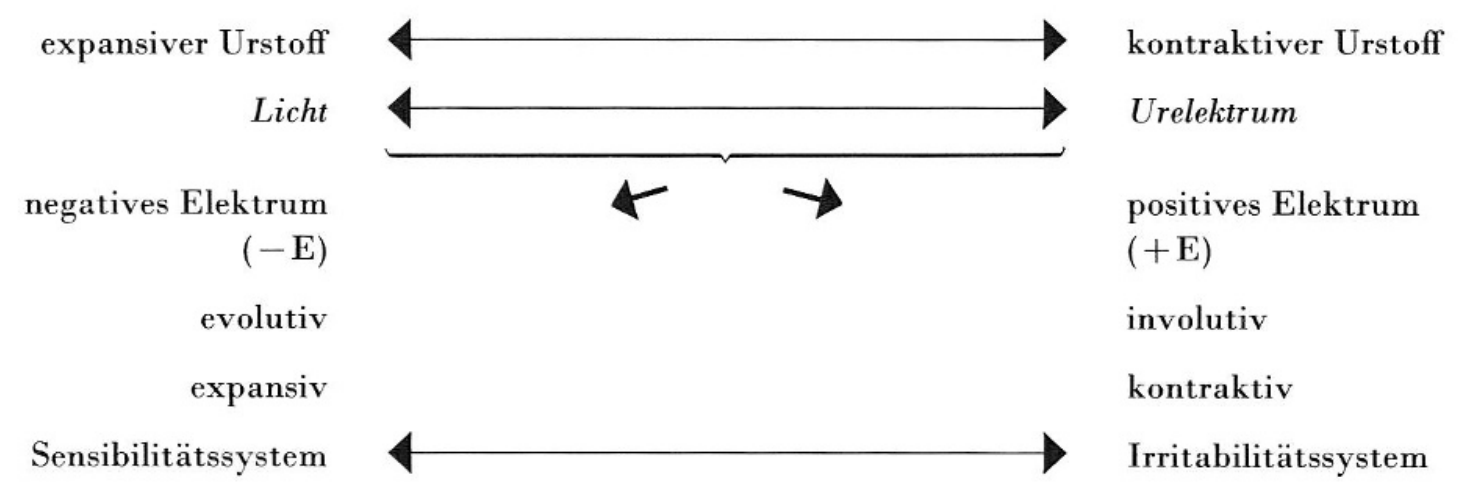

Plastizitätssystem

1. Plastizitätsstufe (Reproduktion/Ernährung)

a) niedrige Organisationsstufe (annäherndes Gleichgewicht von Irritabilität und Sensibilität)

b) Vorwaltende Irritabilität (Pflanzen)

c) Vorwaltende Sensibilität (Tiere, Menschen) 
2. Irritabilitätsstufe (reizbares System)

a) Pflanzenirritabilität

b) Tier- und Muskelirritabilität

- Arterien: kontraktiv

- Venen: evolutiv ${ }^{25}$

3. Sensibilitätsstufe (empfindungsfähiges System)

Perfektionslinie vom Tier bis zu dem Menschen

Nervensystem:

a) Vorwalten des expansiven Faktors in der markigen Nervensubstanz (-E)

- Hirn

- Rückenmark

- Ganglien

b) Vorwalten des kontraktiven Faktors in der fibrös-faszikulären Nervensubstanz (+E)

- Nerven

\section{Theorie der Krankheit}

«Das Individuum ist in Ansehung desselben auf eine gewisse Grenze eingeschränkt, welche nicht überschritten werden kann, ohne sein Bestehen als Produkt unmöglich zu machen: es ist dadurch der Krankheit unterworfen. Die Construktion dieses Zustandes ist ein nothwendiger Theil der allgemeinen Naturlehre, und von dem, was man Physiologie genannt hat, nicht zu trennen.» ${ }^{26}$

Im Folgenden soll nun belegt werden, daß Harless aus seiner naturphilosophischen Konzeption der Physiologie im Sinne dieses Schelling-Zitates auch seine Theorie der Krankheit entwickelte.

1805 stellte Schelling in den Jahrbüchern der Medicin als Wissenschaft eine neue Definition der Krankheit auf: Er definierte die drei Potenzen Reproduktion, Irritabilität, Sensibilität entsprechend seiner real-idealen Naturkonzeption als Dimensionen, wobei jede Dimension vorrangig qualitativ bestimmt wurde, jedoch auch ein bestimmtes quantitatives Verhältnis der beiden Faktoren bedingte. ${ }^{27}$

Allgemein definierte er Krankheit als einen Konflikt des Verhältnisses der Erregung mit der Metamorphose. Hier ist unter Erregung die Erscheinung des inneren, qualitativen Verhältnisses zu verstehen, d. h. durch dieses bestimmt und nicht etwa für dieses bestimmend. Dagegen ist der Erregungsgrad nur eine quantitative Beziehungsgröße des Verhältnisses zwischen Organismus und Außenwelt. ${ }^{28}$ 
Sobald also der Grad der Erregung so groß oder so klein wird, daß dadurch die innere, d.h. qualitative Bestimmung des Organismus im Ganzen oder im Einzelnen verändert wird, ist auch die Identität des Organismus mit sich selber aufgehoben. Das bedeutet eine Veränderung der Norm: Krankheit. Vor allem aber bedeutet das übermäßige Hervortreten einer bestimmten Dimension eine qualitative Veränderung der Norm: «... so macht jede äußre Einwirkung an den Organismus im Ganzen und Einzelnen unmittelbar die Forderung einer bestimmten Dimension (z. B. der Irritabilität oder der Reproduktion), und dieses ist die einzige Wirkungsweise, welche äußre Ursachen überhaupt auf ein innerlich abgewogenes, qualitativ gegliedertes und in sich geschlossenes, mit einem Wort organisches Ganzes ausüben können.» (Jahrb. 187)

Harless' Ableitung des Krankheitsbegriffes aus seinem System der Physiologie ist dieser Dimensionstheorie fast gleich: Durch einen zu sehr gesteigerten oder verminderten Erregungsgrad tritt in einem System einer der bisher gebundenen Faktoren vor den im Normalzustand (Gesundheit) dominierenden: «... und will man bezeichnen ..., daß unter gewissen Momenten der im Normalzustand gebundene und untergeordnete Mitfaktor sogar eine größere und freiere Gewalt erlangen, und dadurch das System gleichsam der Natur des entgegengesetzten mehr annähern könne, wenn man sagt, in der Sensibilität (d.h. der organisch modificierten) werde das irritable Moment ... hervorgerufen, oder in der Irritabilität das sensible Moment.» (Handb. I 61)

Als besonders eindrucksvolles Beispiel dieser Theorie sei hier noch der bei Harless beschriebene Sensibilitätstod genannt: Bei einem zu starken Heraustreten der Sensibilität - Sensibilität wird durch äußere Einwirkung zu stark entzogen -, ist entweder ein Zurücksinken auf den dann stärker werdenden kontraktiven Faktor (Irritabilität) oder, im Fall einer sehr plötzlichen und übermäßigen Entziehung, der Tod die Folge: «Und wenn die Sensibilität in ihnen (Arten dieser Stufe) allen zu stark und zu frei entwickelt wird, wenn sie dadurch in einen ihrem Urwesen näher kommenden Zustand versetzt wird, und in diesem von dem gleichmäßig freieren Gegenpol der äußeren Welt zu stark und im Übermaß angezogen, somit dem Gebilde zu reichlich entzogen wird, so sinkt das Gebilde entweder in Folge des in ihm dann stärker, ja überwiegend werdenden kontraktiven Faktors wieder auf die Stufe des rein negativen Lebens und der simplen Kontraktilität zurück, oder es wird, im Fall der plötzlichen und absolut übermäßigen Entziehung seines organischen Sensibilitätsgehaltes durch äußere Kräfte, sein eigen- 
thümliches Leben sammt seiner Form ganz zerstört. Es stirbt den Sensibilitätstod, welchen allein alle Geschöpfe dieser Klasse früher oder später erleiden.» (Handb. 69 f.)

So zeigt sich hier zum Schluß unter anderem auch an der Theorie der Krankheit, in der Harless sogar die Schellingsche Dimensionstheorie übernommen hat, die ansonsten keine weiteren Auswirkungen auf medizinische Konzepte hatte, daß er zu den konsequenten Vertretern der Schellingschen Naturphilosophie gehörte.

\section{Anmerkungen}

1 Zu den Einzelheiten: M. Lenz, Geschichte der königlichen Friedrich-Wilhelms-Universität zu Berlin, I-IV, Halle 1910; F.v.Bezold, Geschichte der Rheinischen FriedrichWilhelms-Universität, Bonn 1920, I 70.

7 W.Erman, Der tierische Magnetismus in Preußen vor und nach den Freiheitskriegen aktenmäßig dargestellt, München 1925, 5 f.

3 Eine biographische Darstellung findet sich bei: J.F.Hermann, Christian Friedrich Harless. Eine biographische Skizze, Berlin 1857 (mit vollständigem Schriftenverzeichnis) 1-51; vgl. J.Steudel, Zur Geschichte der Medizinischen Universitäts-Poliklinik Bonn, Bonn 1971, $6 \mathrm{ff}$., und E. Stangenberg, Die Entstehung der medizinischen Poliklinik zu Bonn, Diss. Bonn 1966, 15 ff.; vgl. auch Ch.Triebel-Schubert, Johann Christian Friedrich Harless, in: 150 Jahre Rheinische Friedrich-Wilhelms-Universität zu Bonn 1818-1960, Medizin. Fakultät (erscheint demnächst), wo v.a. Harless' Tätigkeit in Bonn und die Spannweite seiner Schriften dargestellt werden.

4. Anders J.Steudel a. O. (Anm. 3), der sich als einziger bisher um eine Einordnung des Harlessschen Werkes bemüht hat.

5 J.Chr.F.Harless, Der Republikanismus in der Naturwissenschaft und Medicin und unter der Aegide des Eclecticismus, Bonn 1819 (im Folgenden zitiert als Harless, Rep.), wo H. Eklektizismus v. a. gegenüber dem von ihm kritisierten Synkretismus absetzt; auf diesen Aufsatz bezieht Steudel seine Einordnung des Harlessschen Standpunktes innerhalb des Eklektizismus, ohne jedoch im Einzelnen darauf einzugehen, wie H. diesen Eklektizismus definiert. Vgl. a. N.Tsouyopoulos, Andreas Röschlaub und die romantische Medizin, Stuttgart 1982, 154ff., die sich um die theoretische Eingrenzung einer Gruppe von Eklektikern bemüht, als deren Hauptvertreter sie Hufeland nennt. Sie betont, wie groß die Differenzen zwischen diesen Eklektikern und den naturphilosophisch orientierten Medizinern waren.

6 In diesem Sinn äußert sich J.Steudel a. O. (Anm.3).

7 J.Ch.F.Harless, Über und gegen den neueren Empirismus in der Physiologie und Medicin, Rhein. Jb. f. Medicin u. Chirurgie 2 (1820) $12 \mathrm{ff}$. (im Folgenden zitiert als Harless, Emp.); vgl. Harless, Rep. $31 \mathrm{f}$.

8 Harless, Emp.51f. 
9 Harless, Rep. $31 \mathrm{f}$.

10 Harless, Emp. 12 f.; vgl. zur dialektischen Triade und der Ableitung der grundlegenden Prinzipien: W. Hartkopf, Schellings Naturphilosophie, Philosophia Naturalis 17 (1979) 349 ff.; vgl. a.: ders., Die Dialektik in Schellings Ansätzen zu einer Naturphilosophie, Monogr. zur philosoph. Forschung Bd. 102, 1972.

11 J.Chr. F. Harless, Handbuch der ärztlichen Klinik, Bd. I: Leipzig 1817, Bd. II: Koblenz 1824, Bd.III: Koblenz 1826. Vgl. für die Physiologie: I 25 ff. (im Folgenden zitiert als Harless, Handbuch I).

12 Harless, Handbuch I 32; vgl. zur Materiededuktion: F. W. J.Schelling, Erster Entwurf eines Systems der Naturphilosophie, Jena/Leipzig 1799 = ND Darmstadt 1981, Schriften 1799-1801, $169 \mathrm{ff}$; ders., System des transzendentalen Idealismus 1804 = ND Darmstadt 1982, Schriften 1801-1804, $440 \mathrm{ff}$.

Der Vergleich mit Schelling bezieht sich hier auf die späteren Ausführungen im System des transzendentalen Idealismus, da die nochmalige Aufnahme der naturphilosphischen Problematik in der (Allgemeinen Deduktion) zu einem Abschluß der eigentlichen Naturphilosophie geführt wird.

Vgl. auch K. E. Rothschuh, Physiologie, München 1968, $191 \mathrm{ff}$.

13 F. W. J.Schelling, System des transz. Ideal. a. O. (Anm. 12) 44.3: «So sind auch die durch die gemeinschaftliche ... Grenze geschiedene Repulsiv- und Attraktivkraft ... nur die Faktoren zur Construktion der Materie, nicht das Construierende selbst.» Zitat im Text: Handbuch I 33 .

14 Schelling, System des transz. Ideal. a. O. (Anm. 12) $444 \mathrm{ff}$.

15 Harless beschreibt die Entwicklung dieses Prozesses parallel dazu auch mit Analogien: der expansive Urstoff entspricht dem Licht (Lichtstoff), der kontraktive Urstoff dem Massenelement, dem Urelektrum. Durch die Verbindung von Urelektrum und Licht entstehen die Urstoffe aller Massen, das positive und das negative Elektrum (Handbuch I 33 f.).

16 Auch Harless sieht die Bildung von Organismen als einen Individualisierungsprozeß, der sich aus dem Gegensatz von Urtätigkeit (Urleben als evolutives Prinzip) und Urkontraktion (Urform der Organismen als kontraktives Prinzip) entwickelt. Hier betont er ebenso wie Schelling die genetische Methode der Naturgeschichte, d.h. der Naturentwicklung.

17 Harless, Handbuch I $50 \mathrm{ff}$.

18 Harless, Handbuch I 51.

19 Schelling, Vorlesungen über die Methode des akademischen Studiums, 13. Vorlesung: Über das Studium der Medicin und der organischen Naturlehre überhaupt, $1803=$ ND Darmstadt 1982, Schriften 1801-1804, 575 f. vgl. ders., Vorläufige Bezeichnung des Standpunktes der Medicin nach Grundsätzen der Naturphilosophie, Jahrbücher der Medicin als Wissenschaft, Tübingen 1805, $165 \mathrm{ff}$.

Vgl. dazu auch N.Tsouyopoulos a. O. (Anm.5) $164 \mathrm{ff}$., die belegt, wie diese Änderungen der Schellingschen Ideen mit seinem Bemühen zu verbinden sind, ein einheitliches System der Naturentwicklung zu schaffen, in dem die gesamte Natur als ein Organismus dargestellt wird. Daraus sollte nicht nur die Physiologie, sondern auch die Entstehung der Krankheit zu entwickeln sein.

Zu der Frage, inwieweit die Naturphilosophie in ihrer späteren Form bei Schelling Differenzen zu der früheren Form zeigt: W.Hartkopf, Schellings Naturphilosophie, 
Philosophia Naturalis 17 (1979) 368, der in der späteren, in die Identitätsphilosophie einbezogenen Naturphilosophie bloße Strukturtheorie sieht.

$\mathrm{Zu}$ den persönlichen und inhaltlichen Auseinandersetzungen zwischen Schelling und Röschlaub, die diese Änderung der Konzeption begleiten: Tsouyopoulos a.O.

20 Schelling, Erster Entwurf (Anm.12) 169 ff.; vgl. dazu E. Mende, Die Entwicklungsgeschichte der Faktoren Irritabilität und Sensibilität in deren Einfluß auf Schellings «Prinzip» als Ursache des Lebens, Philosophia Naturalis 17 (1979) $327 \mathrm{ff}$.

21 Vgl. Anm. 19.

22 Schelling, Jahrbücher 170 (Anm. 12).

23 Harless, Handbuch I 45 f., vgl. dazu oben S. 150 mit Anm.9; dieser Gedanke zeigt konkret, wie die oben dargelegte Konzeption der methodischen Übertragung von konstitutiven Grundsätzen aus dem spekulativen Bereich in die allgemeine Physiologie durchgeführt wird.

24 Vgl. dazu Harless, Handbuch I 57-91.

25 Hier erläutert Harless, Handbuch I $80 \mathrm{f}$. am Beispiel des Herzens, wie er sich dieses antagonistische Verhältnis zwischen Venen und Arterien als Repräsentanten des kontraktiven und expansiven Prinzipes vorstellt. V.a. betont er, daß es sich hier (zwischen Venen und Arterien) nicht um einen unbedingten Gegensatz handele, da doppelt-dynamische Übergänge vorhanden seien: Von den beiden Kammern des Herzens (Aortenkammer, Lungenkammer) besitzt die Aortenkammer die höchste Irritabilität, während die Lungenkammer nur Venenirritabilität besitzt, d.h. eine ganz niedrige Irritabilität, die schon mehr dem Nervensystem (=Sensibilitätsstufe) zugewandt ist. In dieser Polarität sieht er die Ursache des Herzschlages. Dagegen nähert sich diese polarische Differenz in den beiden Vorkammern fast einer Indifferenz. Vorbereitet wird dieser, einem Gleichgewicht nahekommende Zustand durch das Lungengefäßsystem, das in seinem dynamischen Charakter im Verhältnis zu dem Herzen umgekehrt orientiert ist.

26 Schelling, Vorlesungen 574 (Anm. 19).

27 Schelling, Jahrbücher $180 \mathrm{ff}$. (Anm. 19).

28 Das bedeutet, daß nicht mehr, wie ursprünglich auch von Schelling in Anlehnung an Röschlaub im Ersten Entwurf (vgl. oben Anm.12) vertreten, die Veränderung der Reaktionsfähigkeit (= quantitative Bestimmung in dem Begriff der Erregbarkeit: Ausdruck des Verhältnisses zwischen Rezeptivität und Widerstandskraft bzw. Energie des Organismus) als ursprünglicher Faktor der Entstehung von Krankheit bestimmt wird, sondern das veränderte Verhältnis der drei Grundformen des organischen Lebensprozesses (Reproduktion, Irritabilität, Sensibilität = qualitative Bestimmung. Vgl. N.Tsouyopoulos a.O. (Anm.5); zur Erregbarkeit: K.E. Rothschuh, Konzepte der Medizin, Stuttgart 1978, 394f.

29 Schelling, Jahrbücher 187 (Anm.19); er fügt hinzu, daß äußerliche und innerliche Wirkung nie zu trennen sind, jedoch kein äußerlicher Einfluß unmittelbar im Organismus Asthenie oder Hypersthenie setzt, sondern nur das entschiedenere Hervortreten einer Dimension vor einer anderen (der Irritabilität vor der Sensibilität und umgekehrt). 


\section{Summary}

Johann Chr. Fr. Harless (1773-1853) was one of the typical followers of the german concept «Naturphilosophies and strongly influenced by Friedrich Schelling. This is shown by his concept of physiology. His system is based on the dynamic polarity of powers by which he develops the definition of matter ('Materie') in the same way as Schelling. The organic process is defined by three systems: irritability, sensibility and plasticity. In his theory of illness he follows Schelling strictly by applying Schelling's later concept of dimensions.

Charlotte Triebel-Schubert

Medizinhistorisches Institut

Sigmund-Freud-Straße

5300 Bonn-Venusberg 